\title{
Playing in three makes it simpler: Mapping the cognitive figure-ground framework onto cancer-immunology and immunotherapy (Review)
}

\author{
YORI GIDRON $^{1,2^{*}}$ and LUCA VANNUCCI ${ }^{3,4^{*}}$ \\ ${ }^{1}$ Faculty of Medicine and Pharmacy, Vrije Universiteit Brussel, Laarbeeklaan 103, 1090 Jette, Belgium; \\ ${ }^{2}$ IESEG School of Business, Rue de la Digue 3, 59000 Lille, France; ${ }^{3}$ Department of Immunology and \\ Gnotobiology, Institute of Microbiology, Academy of Sciences of the Czech Republic, v.v.i., Videnska 1083, \\ 14220 Prague 4; ${ }^{4}$ Laboratory of Tumour Biology, Institute of Animal Physiology and Genetics, \\ Academy of Sciences of the Czech Republic,v.v.i., Rumburská 89, 27721 Libechov, Czech Republic
}

Received September 18, 2009; Accepted November 17, 2009

DOI: 10.3892/ijo_00000587

\begin{abstract}
The avalanche of research findings in complex multidisciplinary fields, such as cancer immunobiology, requests organizing and practical working models for scientists and clinicians. Frameworks from one scientific discipline can be adopted for another one, to clarify and provide new insights into complex findings. A 'figure-ground' (FG) perspective was adopted from cognitive sciences to construct a simple organizing tool, which can assist in understanding tumour development and immunotherapy designing. In an FG arena, there is a figure that needs to be contrasted from a background to be seen by a viewer, who may have a mental representation of the figure (i.e. knows what the figure features look like). Applying this framework to cancer, three players emerge: the viewer (immune system components), the figure (tumour), and the background (e.g., normal cells) with their dynamic interactions. Various characteristics of tumour development such as reduced expression of major-histocompatilibity complex (MHC) molecules or infiltration by inflammatory cells in its boundaries make tumour-immunity interplay highly suitable to an FG perspective. We describe the basic FG frame-work and immuno-biology of tumour development, thereafter reframed by the FG freamework. The term 'antigenic contrast' is introduced to reflect the contrast between the tumour figure and its variable background. Antigenic contrast emerges as a main factor enabling the
\end{abstract}

Correspondence to: Dr Yori Gidron, Faculty of Medicine and Pharmacy, Vrije Universiteit Brussel, Laarbeeklaan 103, 1090 Jette, Belgium

E-mail: yori.gidron@vub.ac.be

* Contributed equally

Key words: immune system, cancer development, tumour escape, immunotherapy, cognitive-perceptual, figure-ground immune system viewer to detect and mount adequate reactions against a tumour figure. We provide empirical examples of immunotherapeutic interventions whose results are explained by the FG perspective. For example, vaccines are forms of sharpening the 'mental' representation of the immune viewer concerning the tumour figure, while administering interferons can be seen as enhancing tumour figure salience by rescuing MHC expression. This framework highlights important elements in complex networks (e.g., cancer immunobiology), enhances communication between cancer scientists and clinicians, explains experimental and clinical study results, and provides further rationale for combinational immunotherapies.

\section{Contents}

1. Introduction

2. Parallels between immunity and cognitive systems

3. The figure-ground framework

4. A current view of tumour development and anti-tumour immunity

5. Reframing tumour-immunity interplay by the figure-ground framework

6. Antigenic contrasting of the tumour figure from the inflammatory background

7. Empirical examples of immunotherapeutic figure-ground interventions

8. Conclusions and perspectives

\section{Introduction}

Due to the avalanche of research findings in complex multidisciplinary fields such as cancer biology, scientists and clinicians need organizing and practical working models. This may be possible by adopting paradigms from other sciences. With this purpose in mind, we propose here the figure-ground framework adopted from cognitive-perceptual sciences to 
review experimental findings and clinical research results in cancer-immunology.

\section{Parallels between immunity and cognitive systems}

Cohen suggested viewing the immune system as a cognitive system (1). Both systems are characterized by priming, memory and 'mental representations'. In cognitive neuroscience, priming refers to a first exposure of the perceptual system to a stimulus, which facilitates later responses upon re-exposure to such a stimulus (2). The neural system creates assemblies of associated neuronal units, reflecting cognitive schemas of what general features of a concept/object look like: the mental representations. Memory activation is manifested by the associated neural units becoming active upon re-exposure to a previously presented triggering stimulus.

In immunity, priming involves a first efficacious contact between immune cells and an immunogenic antigen. This implies specific antigen recognition by pre-existing receptors expressed on adaptive immunity cells ( $\mathrm{T}$ and B lymphocytes). Part of the activated cells will target the antigen, while another part will differentiate into memory cells, to establish a faster specific immune response during future re-exposures to the priming antigen. Finally, the panel of memory cells and antigen receptors can be considered as the immune system's 'mental representation' of the antigenic environment it has been progressively exposed to (1). These parallels are crucial for viewing cancer-immunity networks in a cognitive-perceptual perspective, we now introduce.

\section{The figure-ground framework}

In cognitive-perceptual systems, a viewer is able to distinguish an object (figure) from its background. The degree of spatial impenetrability of elements in a picture, and the process of feedback between mentally represented boundaries (what things look like) and actually observed surfaces, help to distinguish a figure from the ground (3). Mapping this paradigm onto immunological contexts, the figure represents the immunological target (e.g., tumour cells) which the viewer (immunity) can detect and attack, depending on the figure's contrast from its background (the context in which a tumour develops). The contrast may increase by enhancing the figure's visibility, shadowing the background, or by sharpening the viewer's mental representation of the figure.

\section{A current view of tumour development and anti- tumour immunity}

Current models of tumorigenesis shift from a cellular centred approach (cell transformation model) to focussing on tumour microenvironment and local inflammation (4-6). The tumour microenvironment includes the transformed cells and their products, the normal tissue cells, stroma, vessels, nerves, immune cells and all related products. When transformed cells become immortal and non-responsive to tissue homeostatic control (7), they begin to multiply, eliciting danger signals [e.g., heat-shock proteins, interleukin (IL)-1ß, interferons IFNs] (8). This activates a local acute inflammatory reaction, sustained by surrounding innate immunity cells (e.g., natural killer cells, macrophages) $(9,10)$. Antigens from killed tumour cells are then processed by dendritic cells (DCs), and become bound to major histocompatibility complex (MHC) class I molecules. Together, they are presented to and prime cytotoxic T lymphocytes (CTLs). Activated CTLs specifically target the tumour, until eradication. This process also generates specific memory cells. However, several escape mechanisms (e.g., tumour down-regulated expression of MHC class I molecules (11); immunoediting and immunosculpturing (12), permit mutated cells to avoid immune surveillance and to multiply (13). This growth produces further stress on surrounding tissues. The newly elicited stress signals further sustain inflammatory responses, and the initially defensive, local and acute inflammation becomes chronic (14). Homeostatic antiinflammatory and immunosuppressive mechanisms are stimulated (T helper type 2 - Th2; T regulatory cells - T-regs), and by suppressing CTLs, they paradoxically contribute to cancer progression (15).

\section{Reframing tumour-immunity interplay by the figure- ground framework}

To reframe the described and very complex dynamics of tumour development by the figure-ground $(\mathrm{F}-\mathrm{G})$ perspective, we need to identify three players: figure, background and viewer. At the beginning, the transformed cells represent a figure situated inside the background of a self context - normal tissue (Fig. 1). The viewer is immunity and its components. It changes locations and functions during tumour development, as following.

Stage 1: Initially, when cells transform, their 'non-self' antigenic pattern may be insufficiently 'strong' to be distinguished by the immune viewer from the surrounding normal 'self' tissue (background). The viewer here is represented by nearby cells of innate immunity. Once danger signals are elicited by multiplication of transformed cells, the transformed cell figure achieves 'perceptual' (immunological) salience for the viewer. This triggers the viewer's activation (immune cell migration and cell-mediated killing) (Fig. 1).

Stage 2: Tumour antigens are taken up and processed by antigen-presenting cells (APC: macrophages and DCs). DCs, migrating to lymph nodes, prime the second, more potent, viewer - CTLs. This activated second viewer (effector cells) specifically identifies the tumour figure for its eradication. This process enlarges and 'sharpens' the viewer's mental representation of the tumour figure (Fig. 2). However, if the activated immune viewer fails to eradicate the tumour, transformed cells continue to replicate and elicit inflammatory signals, maintaining the tumour figure locally visible.

Stage 3: Expansion of the tumour and release of danger signals continues to stimulate recruitment of immune cells to surround, infiltrate, and join the tumour figure (Fig. 3A and B). The creation of the tumour microenvironment (including the tumour cells, the stroma and the previously activated local inflammatory network) produce changes in the original tumour figure, making the contrast between the tumour and its background weaker. Recruited immune cells include the first viewer (local innate-immunity cells) and the second viewer (CTLs). Progressively, the inflammatory network inside and near the tumour shadows the original tumour figure. 
$\mathbf{A}$

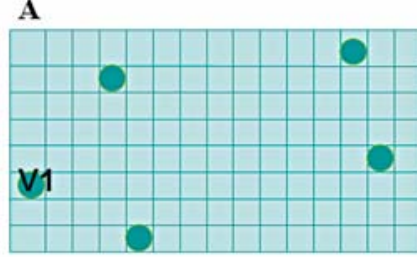

C

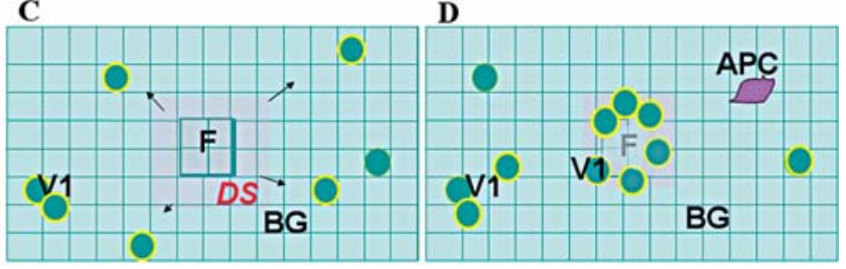

Figure 1. Figure-ground model applied to normal immune response against a tumour: identification of the figure. (A) Normal tissue represents the background (BG). (B) Initially transformed cells create a figure (F). This figure does not create enough contrast on the background since the 'self' antigenic pattern is not yet deeply mutated. (C) Enhancement of the figure visibility is permitted by elicitation of danger signals (DS), attracting and inducing activation of the local immune cells that represent the first viewer (V1). (D) The activated V1 targets the enhanced figure for is ablation.

$\mathbf{A}$

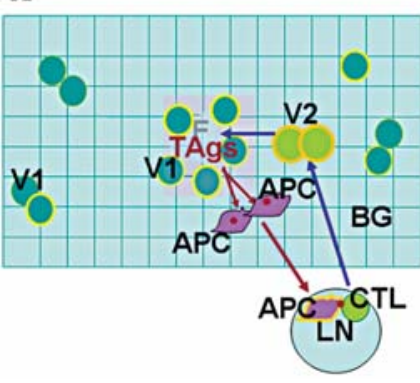

B

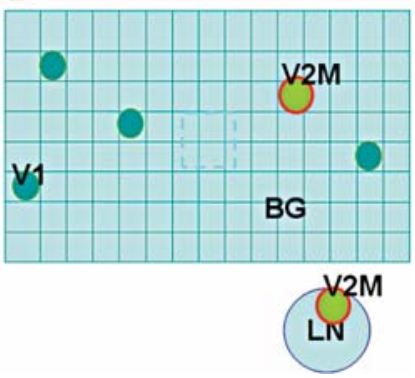

Figure 2. Figure-ground model applied to normal immune response against a tumour: elimination of the tumour-figure. (A) The killing of transformed cells by V1 makes tumour antigens (TAgs) to be available for up-taking by antigen presenting cells (APC), i.e., macrophages and dendritic cells. The dendritic cells carrying elaborated TAgs (brown dots) migrate in the lymph nodes to mature and actively present the TAgs to cytotoxic T lymphocytes (CTL). Specifically primed and activated, the CTLs become the second viewer (V2), with selective activity against the tumour figure. (B) The tumour ablation by V2 leads to reconstituted homeostasis in the tissue. Part of the specifically primed CTLs differentiates as memory cells (V2M), ready to be activated if a new challenge by the priming TAg recurs.

Stage 4: Persistence of inflammation triggers the response of a third viewer: systemic immunity (Fig. 3C). Its 'attention' is attracted to the inflammatory tumour microenvironment figure. Therefore, the chronic inflammatory network is seen as 'THE' homeostatic problem, instead of the tumour. Thus, systemic immunity mechanistically activates physiological anti-inflammatory regulatory responses (Th2, T-regs). This suppresses CTLs and other tumour-aggressing cells, and together with tumour-derived immunosuppressive molecules, contributes to tumour progression (14).

\section{Antigenic contrasting of the tumour figure from the inflammatory background}

Introducing the term 'antigenic contrast', we refer to the immunological salience obtained by the tumour cell antigenic

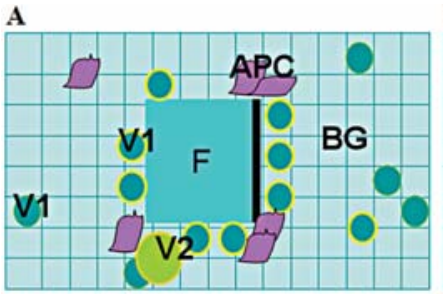

B

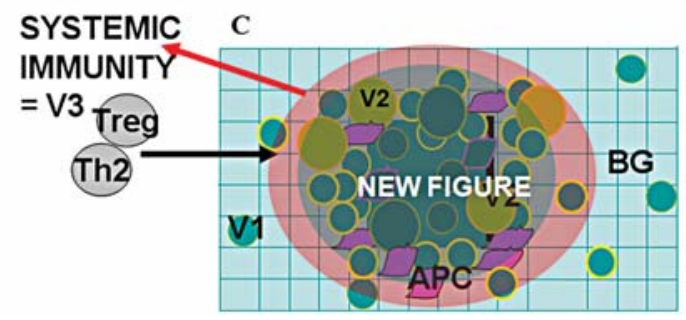

Figure 3. Figure-ground model applied to tumour escape: modification of the figure and misled involvement of a new viewer. (A) Progressing tumour figure elevates from background and stimulates persistence of immune targeting by V1 and V2. (B) Continuous activation and recruitment V1 and V2 from the background (normal tissue surrounding the tumour). V1 and V2 surrounding and migrating inside the tumour figure sustain the local inflammation that become chronic. Chronic inflammation and infiltrated immune cells (V1 and V2) progressively shadow and modify the tumour figure. (C) A predominantly inflammatory figure is formed, composed of tumour cells, tumour infiltrating and tumour surrounding immune cells (V1, V2 and APC), and pro-inflammatory molecules. This new figure, more complex than the original one, elevates on the surrounding normal background (BG) for its inflammatory characteristics and goes to trigger the intervention of a third viewer (systemic immunity, V3). The V3 is induced to mount a regulatory response [by $\mathrm{T}$ helper 2 (Th2) and $\mathrm{T}$ regulatory (T-reg) cells] to re-establishing the tissue homeostasis (termination of the chronic inflammation). This can paradoxically favor tumour escape (see text).

phenotype (original figure and true target) relative to its background (inflammatory microenvironment or surrounding tissue). As described above, this contrast may decrease with tumour development. We hypothesize that adequate 'antigenic contrast' could rescue effective identification of the tumour figure in its obscuring background. Consequently, the F-G framework predicts that interventions aimed at increasing the antigenic contrast between the tumour figure and its inflammatory parts or its inflammatory background, should be done, for example, by: a) enhancing expression of tumour associated antigens (TAA) or inducing re-expression of MHC class I molecules on tumours (increasing tumour salience); b) 'shadowing' (suppressing) the inflammatory network; c) sharpening the viewer's mental representation of the tumour figure using vaccines.

\section{Empirical examples of immunotherapeutic figure- ground interventions}

The role of the inflammatory background in an FG perspective can be easily seen when comparing germ-free (GF) with conventionally $(\mathrm{CV})$ reared animals. In CV conditions, the commensal microbiota induces a highly regulated inflammatory network in the gut, known as 'chronic physiological inflammation' - CPI (16). Immune tolerance is induced, to avoid inflammatory damage of the mucosa. However, GF animals lack CPI and its elevated regulatory network (e.g., TGF-B, IL-4, IL-10). Following induction of colorectal 
cancer, GF animals developed less and smaller tumours, and maintained higher anti-tumour immunological activity than $\mathrm{CV}$ animals (more cytotoxic cells, greater cytotoxicity, and more B cells). GF animals thus have a constitutively lower inflammatory background and are immunologically more 'naive' (they had more limited challenge by intestinal antigens, to be tolerated) than in $\mathrm{CV}$ conditions. These may have permitted greater salience of the tumour figure (adequate 'antigenic contrast'), and reduced shadowing during progression in GF animals (17).

Use of anti-inflammatory drugs (e.g., COX-2 inhibitors, aspirin) to prevent colorectal cancer (18) can be interpreted as shadowing the inflammatory background. On the other hand, experimentally administering IFNs or histone deacetylase inhibitors (e.g., Trichostatin A) to rescue MHC class I expression $(19,20)$, and tumour-cell engineering to increase immunogenic molecule expression on cell surfaces (21) may enhance figure salience. Vaccination, e.g. with TAA, DNA or DC vaccines, can sharpen the viewer's 'mental representation' of tumour figures (tumour antigenic phenotype) (22).

Finally, the FG framework also provides a strong rationale for combinational treatments: sharpening mental representations by vaccines can be insufficient if the tumour figure is too weak or if it is shadowed. Experimental studies in vivo on animal models demonstrated that addition of anti-inflammatory (shadowing) medications enhanced the effectiveness of cancer vaccines (sharpened mental representation) (23). Similarly, administering a vaccine which included IFN $\gamma$ (figure-enhancement by rescued expression of MHC-I), led to stronger anti-tumour responses than the regular vaccine alone (24). Finally, in humans with bladder cancer, aspirin (background shadowing) enhanced patients' disease-free survival following vaccination with bacille Calmette-Guérin (25), a generic immune stimulator. Thus, as predicted by the FG perspective, sharpening the viewer's representation of tumour antigens with vaccines and, either reducing the inflammatory background or enhancing the figure's antigenicity, synergistically interact in favour of tumour eradication.

\section{Conclusions and perspectives}

The application of the FG framework from cognitiveperceptual sciences to the analysis of the tumour-immunity network showed its ability to serve as a tool for highlighting important aspects of a complex bio-medical process. The identification of the three main players inside the model of cancer microenvironment development, permitted to define and clarify the phases of their dynamic relationships and evolution. By doing this, the targets for therapeutic interventions were made more evident. We introduced the concept of 'antigenic contrast' as a major factor that permits the immune system (viewer) to effectively detect and target the tumour figure. Consequently, three possible modalities of intervention were identified to effectively increase the tumour figure salience and its eradication - by shadowing the inflammatory background, by sharpening the viewer's representation of the tumour figure with a vaccine, or by re-establishing correct tumour antigenicity. Importantly, the FG framework also indicates the necessity and provides a rationale for combining these interventions to yield synergistically stronger and more effective anti-tumour responses. This framework appears to be a useful perspective tool to 'catch the crux' of biomedical problems (as tested here), for designing and guiding experimental and clinical approaches, and for enhancing communication between biologists and clinicians. These can all serve future translational research.

\section{Acknowledgements}

We thank the GAAV (CZ) for grant no. IAA500200917, the Institutional Research Concepts no. AV0Z50200510 (ASCR v.v.i., CZ), IRP IAPG no. AV0Z50450515, and Professor Jean Vroomen from University of Tilburg, for critically reading an earlier version of this manuscript.

\section{References}

1. Cohen IR: The cognitive principle challenges clonal selection. Immunol Today 13: 441-444, 1992

2. Tulving E and Schacter DL: Priming and human memory systems. Science 247: 301-306, 1990

3. Grossberg S and Pessoa L: Texture segregation, surface representation and figure-ground separation. Vision Res 38: 2657-2684, 1998.

4. Laconi E: The evolving concept of tumor microenvironments. Bioessays 29: 738-744, 2007.

5. Albini A and Sporn MB: The tumour microenvironment as a target for chemoprevention. Nat Rev Cancer 7: 139-147, 2007.

6. Lorusso $\mathrm{G}$ and Rüegg C: The tumor microenvironment and its contribution to tumor evolution toward metastasis. Histochem Cell Biol 130: 1091-1103, 2008.

7. Hanahan D and Weinberg RA: The hallmarks of cancer. Cell 100: 57-70, 2000

8. Gallucci $\mathrm{S}$ and Matzinger P: Danger signals: SOS to the immune system. Curr Opin Immunol 13: 114-119, 2001.

9. Le Bitoux MA and Stamenkovic I: Tumor-host interactions: the role of inflammation. Histochem Cell Biol 130: 1079-1090, 2008.

10. Whiteside TL: The role of immune cells in the tumor microenvironment. Cancer Treat Res 130: 103-124, 2006.

11. Bubenik J: MHC class I down-regulation: tumour escape from immune surveillance? (Review). Int J Oncol 25: 487-491, 2004.

12. Reiman JM, Kmieciak M, Manjili MH and Knutson KL: Tumor immunoediting and immunosculpting pathways to cancer progression. Semin Cancer Biol 17: 275-287, 2007.

13. Stewart TJ and Abrams SI: How tumours escape mass destruction. Oncogene 27: 5894-5903, 2008.

14. Mantovani A, Allavena P, Sica A and Balkwill F: Cancerrelated inflammation. Nature 454: 436-444, 2008.

15. Lamprecht B, Kreher S, Anagnostopoulos I, Jöhrens K, Monteleone G, Jundt F, Stein H, Janz M, Dörken B and Mathas S: Aberrant expression of the Th2 cytokine IL-21 in Hodgkin lymphoma cells regulates STAT3 signaling and attracts Treg cells via regulation of MIP-3alpha. Blood 112: 3339-3347, 2008.

16. Tlaskalová-Hogenová H, Stepánková R, Hudcovic T, Tucková L, Cukrowska B, Lodinová-Zádníková R, Kozáková H, Rossmann P, Bártová J, Sokol D, Funda DP, Borovská D, Reháková Z, Sinkora J, Hofman J, Drastich P and Kokesová A: Commensal bacteria (normal microflora), mucosal immunity and chronic inflammatory and autoimmune diseases. Immunol Lett 93: 97-108, 2004.

17. Vannucci L, Stepankova R, Kozakova H, Fiserova A, Rossmann P and Tlaskalova-Hogenova H: Colorectal carcinogenesis in germ-free and conventionally reared rats: different intestinal environments affect the systemic immunity. Int $\mathbf{J}$ Oncol 32: 609-617, 2008.

18. Antonakopoulos N and Karamanolis DG: The role of NSAIDs in colon cancer prevention. Hepatogastroenterology 54: 1694-1700, 2007.

19. Yang I, Kremen TJ, Giovannone AJ, Paik E, Odesa SK, Prins RM and Liau LM: Modulation of major histocompatibility complex Class I molecules and major histocompatibility complex-bound immunogenic peptides induced by interferon-alpha and interferon-gamma treatment of human glioblastoma multiforme. J Neurosurg 100: 310-319, 2004. 
20. Manning J, Indrova M, Lubyova B, Pribylova H, Bieblova J, Hejnar J, Simova J, Jandlova T, Bubenik J and Reinis M: Induction of MHC class I molecule cell surface expression and epigenetic activation of antigen-processing machinery components in a murine model for human papilloma virus 16-associated tumours. Immunology 123: 218-227, 2008.

21. Schabowsky RH, Sharma RK, Madireddi S, Srivastava A, Yolcu ES and Shirwan H: ProtEx technology for the generation of novel therapeutic cancer vaccines. Exp Mol Pathol 86: 198-207, 2009.

22. Chen $\mathrm{X}$, Chang $\mathrm{CH}$ and Goldenberg DM: Novel strategies for improved cancer vaccines. Expert Rev Vaccines 8: 567-576, 2009.
23. Haas AR, Sun J, Vachani A, Wallace AF, Silverberg M, Kapoor V and Albelda SM: Cycloxygenase-2 inhibition augments the efficacy of a cancer vaccine. Clin Cancer Res 12: 214-222, 2006.

24. He X, Luo P, Tsang TC, Zhang T and Harris DT: Immuno-gene therapy of melanoma by tumor antigen epitope modified IFNgamma. Cancer Immunol Immunother 54: 741-749, 2005.

25. Gee JR, Jarrard DF, Bruskewitz RC, Moon TD, Hedican SP, Leverson GE, Nakada SY and Messing EM: Reduced bladder cancer recurrence rate with cardioprotective aspirin after intravesical bacille Calmette-Guérin. BJU Int 103: 736-739, 2009. 